\title{
Effectiveness of Bulbous rudder with Horizontal Fins on the Propulsion Performance of Ship in Waves
}

\author{
by Win Naing HTAY*, Student Member Benson Oyunge MWANGI* \\ Hina MATSUURA* Yasuyuki TODA*, Member
}

\begin{abstract}
Summary
Over the years, shipping has been played on one of the most used transportation methods and marine trading is increasing more and more these days. As a result, the shipbuilders are trying to design and construct an efficient and enormous ship to be economical. On the other hand, the environmental awareness to eliminate greenhouse gas emission is rising due to the effect of global warming. Such factors make the various types of energy-saving devices (ESDs) to be developed reliably without affecting other functions. This paper characterizes the performance of the rudder bulb-fins system (RBF) fixed behind the propeller in wave. In this study, the analysis of dynamics forces and velocity fields around the stern which are crucial in evaluating the performance of ESDs were implemented using CFDShip-Iowa V4.5. The effect of the ESD on ship's motion was analyzed by performing motions analysis in various regular head waves. The computational results were validated with the experiments conducted in the towing tank of Osaka University. The calculations were done for three cases of rudder conditions: the ship without a rudder, the ship with conventional rudder, and the ship with RBF. The predicted results and measured data reveal that the currently designed RBF can diminish the hub vortex strength and improve the hull efficiency due to the increase of thrust and wake gain without any adverse conditions. It can be confirmed that the computational simulations predicted and followed the trend of experimentally measured data well.
\end{abstract}

\section{Introduction}

International Maritime Organization (IMO) remains committed to reducing greenhouse gas emissions from international shipping and, as a matter of urgency, focuses on phasing them out as soon as possible in this $21^{\text {st }}$ century. Energy Efficiency Design Index (EEDI) which is the most important technical measure to promote the utilization of more energy-efficient equipment and engines was made mandatory for new ships by IMO. As a result, many ship designers around the world are trying to implement new technologies to improve the efficiency of the vessels. Generally, these new techniques are optimization of hull form, high-efficiency propeller design, low frictional resistance type antifouling coating system, and energy saving devices (ESDs) around the propeller. A variety of ESDs has been invented and applied according to their working principles and location.

Various researches on the performance of ESDs has been carried out experimentally and computationally in recent years. Tacar et al. demonstrated experimentally and numerically the effect of the gate rudder system that improve propulsive efficiency of $14 \%$ in sea trial on the powering performance of a container ship in two different model scales ${ }^{1)}$. Okada et al. executed the study on the development of ultimate rudders with small and large diameter of rudder bulb for bulb carrier numerically in which $3.5 \%$ efficiency improvement at small diameter and $4.1 \%$ at larger diameter were observed $^{2)}$. The interaction mechanism between the hull, pre

\footnotetext{
* Department of Naval Architecture and Ocean Engineering, Osaka University
}

swirl stator and rudder bulb was analyzed by $\mathrm{Su}$ et al. The average efficiency gain of up to $3 \%$ was obtained ${ }^{3)}$. The energy gain predicted at the full scale of ship with rudder bulb and fins was less than that in the model scale ${ }^{4}$. Htay et al. investigated computationally the performance of rudder bulb fins system of KVLCC2 and predicted motions and forces in regular head waves. A small amount of improvement of hull efficiency in certain wave conditions was confirmed ${ }^{5) 6}$.

This paper investigates the effect of the rudder bulb fins systems (RBF) from the aspect of propulsion performance. RBF is mounted behind the propeller and attached to the leading edge of the rudder. The fundamental working mechanism of RBF is that the rudder bulb smoothens the propeller hub vortex and rudder fins produce the additional thrust. By means of this, RBF can boost effectively the propulsive performance of the vessel and achieve the required EEDI.

The energy-saving not only in ideal condition but also in operational conditions with waves is necessary to prove ESDs by international organizations. Moreover, the detailed flow field, the interaction of the hull, propulsor and ESDs must be fully understood. Therefore, the purpose of this study is to contribute to this requirement through Computational Fluid Dynamics (CFD) analysis and Experimental Fluid Dynamics (EFD). In this research, the predications of motions, viscous flow field around propeller, forces and hull efficiency of KVLCC2 without rudder, with normal rudder and with RBF system were examined using CFD to confirm the influence of the rudder and rudder with ESD on the propulsive performance and validation with experimental data was carried out. The RBF system used in this present research has been approved and validated in calm water. Hull efficiency improvement of 3.6\% at CFD and $7.3 \%$ at EFD was examined ${ }^{7)}$. 


\section{Geometry of ship, propeller and rudders}

The tanker named as KRISO very large crude oil carrier two (KVLCC2) was selected as a targeted ship for this study. A $3.2 \mathrm{~m}$ model ship scale of $1 / 100$ was used to predict the hydrodynamic forces, motions, and the viscous flow field around stern at fully loaded conditions in three different regular head waves. Some principle particulars of KVLCC2 full-scale ship and model ship are listed in Table 1. All the computations and measurements were made for model ship condition at $\mathrm{Fr}=0.142$ and $\mathrm{Re}=2.546 \times 10^{6}$ correlating to the average model ship's speed, Vs of $0.7955 \mathrm{~m} / \mathrm{s}$ which is equivalent to the service speed of 15.5 knots.

Four bladed propeller model with a $9.86 \mathrm{~cm}$ diameter was utilized in self-propulsion tests. The propeller used in the experiments rotates in the clockwise direction with the rotational speed of 16.5 rps which is the model point of the ship with the normal rudder in calm water. That is a little different in CFD simulations where the propeller rotational speed was set to $16.3 \mathrm{rps}$ that is the model point of ship with RBF system in calm water. During the self-propulsion experiments, an additional towing force was taken into account to get the self-propulsion point in waves. The number of revolutions was kept for all wave condition because the loading effect to the wake fraction was avoided by keeping a similar loading value. So, the resistance with propeller and thrust was not balanced. It was done to verify the wake fraction changes due to waves at similar loading.

In this study, simulations and experiments were performed in three different rudder conditions (without rudder, with normal rudder, and with RBF). The 3-D perspective view of normal rudder and rudder with RBF system existing at ship stern are shown in Fig.1. On the starboard side fin, the camber is downward to produce the forward force due to the downward flow generated by the propeller. The camber of the fin on the port side is opposite to that on the starboard side.

Table 1 Principle particulars of KVLCC2

\begin{tabular}{|c|c|c|}
\hline Particulars & Full scale & Model scale \\
\hline Length between perpendiculars,L $(\mathrm{m})$ & 320 & 3.2 \\
\hline Maximum beam at waterline $(\mathrm{m})$ & 58 & 0.85 \\
\hline Depth $(\mathrm{m})$ & 30 & 0.3 \\
\hline Draft $(\mathrm{m})$ & 20.8 & 0.208 \\
\hline Block coefficient, $\mathrm{C}_{\mathrm{B}}$ & 0.8098 & 0.8098 \\
\hline Wetted area without rudder $\left(\mathrm{m}^{2}\right)$ & 27194 & 2.7194 \\
\hline
\end{tabular}
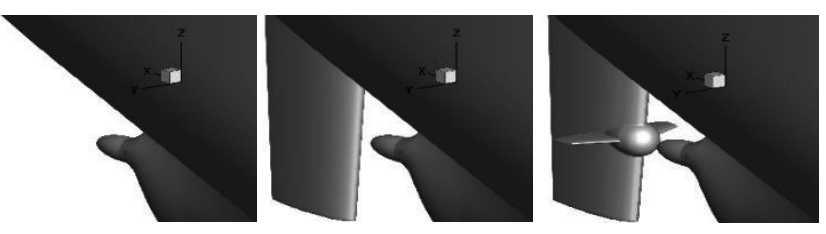

Fig. 1 Ship stern with (left: without rudder, middle: normal rudder and right: RBF)

\section{Methodology}

\section{1 Experimental method}

The experiments were carried out in the towing tank of Osaka University having $100 \mathrm{~m}$ length, $7.8 \mathrm{~m}$ width, and $4.35 \mathrm{~m}$ depth. The ship model was tested in three degree of freedom (DOF) motions which are heave, pitch, and surge. For the surge direction, averaged resistance force was balanced by a weak spring force and constant added force produced by servo motor. So, the surge motion due to exciting force can be measured. The heave, pitch, and surge motions of a model ship in regular linear head waves with $3 \mathrm{~cm}$ of wave amplitude $(\lambda / \mathrm{L}=0.6,1.1$, and 1.6 where $\lambda$ is wave length) were measured using potentiometers which were installed at both fore and aft perpendiculars of the ship. Dynamometers were used to measure the average resistance force (excluding exciting force), thrust, and torque. The SPIV (Stereo Particle Image Velocimetry) measurements were done to visualize the flow field upstream of the propeller and downstream of the rudder in waves. In SPIV measurement, both clockwise and counterclockwise propeller models were used to investigate the velocity field on both sides of the ship since the rudder would block the SPIV laser while measuring the flow field upstream of the propeller. The detailed experimental set up has been stated in the paper of Mwangi et $\mathrm{al}^{8)}$.

\section{2 Computational method}

Nowadays, CFD is widely used in many complicated hydrodynamics applications and it has been used in ESDs research to obtain reasonably good results. CFD can determine the macroscopic forces as well as detailed flow field information. In this study, CFDShip-Iowa V4.5 was applied to solve the hydrodynamics problems. It is an incompressible RANS/DES (detached eddy simulation) solver designed for ship hydrodynamics. The equations are solved in an inertial coordinate system, either fixed to a ship moving at constant speed or in the earth system. The free surface is modelled with a single-phase capturing approach. The 3D level set function, $\phi$, is defined in the whole domain with its value related to the distance to the interface and is set to negative in air, positive in water, and zero at the free surface. The equation for the free surface is as follows ${ }^{9}$.

$$
\frac{\partial \phi}{\partial t}+\frac{\partial\left(\phi U_{i}\right)}{\partial x_{i}}=0
$$

where $U_{i}$ is velocity components and $x_{i}$ is Cartesian coordinates.

To predict the propeller performance, a body force propeller model based on the blade element theory using the CFD output velocity components at the propeller plane was applied ${ }^{10)}$. The propeller blade with radius, $R$ is split into equal sections. The lift $(d L)$ and drag $(d D)$ of each blade element are computed from the resultant velocity at each radial segment which is calculated using Eq. (2) and angle of attack based on total velocity.

$$
U_{r}=\sqrt{(u)^{2}+\left(2 \pi n r-U_{t}\right)^{2}}
$$

where $u$ is the axial velocity including induced velocity in CFD code, $U_{t}$ is the tangential velocity and $n$ is the non-dimensional propeller revolution rate. The thrust $(d T)$ and torque $(d Q)$ at each blade element are calculated from the drag and lift on each blade element and averaged at a fixed point from the moving blade and small cord length approximation. The body forces acting in radial and angular direction on the propeller plane $\left(f_{b x}, f_{b \theta}\right)$ are computed by Eq. (3) and Eq. (4) based on the average effective of $d T$ and $d Q$ among $N$ blades, propeller rotation perimeter $2 \pi r$ at certain radius $r$ and propeller thickness $\Delta x$.

$$
\begin{aligned}
& f b_{x}=\frac{d T \cdot N}{\Delta x \cdot 2 \pi r} \\
& f b_{\theta}=\frac{d Q \cdot N}{\Delta x \cdot 2 \pi r^{2}}
\end{aligned}
$$


The overall thrust $(T)$ and torque $(Q)$ of the rotor are obtained by integrating the individual contribution of area of each element along the radius of the propeller using Eq. (5) in which $R_{B}$ means the radius of propeller boss. Those body forces are used in solving the momentum equations in the URANS solver as source terms.

$$
T=\int_{0}^{2 \pi} \int_{R_{B}}^{R} f b_{x} \Delta x r d r d \theta, Q=\int_{0}^{2 \pi} \int_{R_{B}}^{R} f b_{\theta} \Delta x r d r d \theta
$$

Shear-stress transport (SST) $k-\omega$ turbulence model without wall function which is robust and applicable to complex geometries and flows was used as a turbulence model for closure. A projection solver was implemented to obtain a pressure equation and satisfy continuity. The equations were discretized using a finite differences approach. The second-order upwind scheme was used to discretize the convection term of momentum equation and level set convection term. The non-dimensionalized time step was set to 0.005 and 6000 time steps were considered in these computations. To solve the system of discretized governing equation two or four inner iterations were used in each time step. All the simulations were performed for ship-fixed frame system parallelization with MPI-based domain decomposition. The entire grid system was decomposed into 32 blocks using one processor per block.

\section{2. 1 Grids}

The structured grids with the multi-block overset technique were generated in Gridgen software. In the SUGGAR library, the overset interpolation information between each block was determined. The whole ship body grid system was mainly composed of seven blocks in a ship without rudder, nine blocks in a ship with a conventional rudder, and thirteen blocks in a ship with RBF. The background orthogonal grid was created to cover up the entire ship. A refinement orthogonal grid was designed to be able to capture the better flow field resolution around the ship's stern. Fig. 2 describes the overset grid of ship stern with RBF. The nondimensionalized grid spacing normal to the hull surface was set to $10^{-6}$. The average grid points were nearly 80 points per wavelength along the $\mathrm{x}$-axis and 15 points in one wave amplitude along the $\mathrm{z}$ axis. The total number of grid points was roughly 8 million points in the case of a ship without a rudder, 8.3 million points in a ship with a normal rudder and 9 million points in a ship with the RBF respectively.

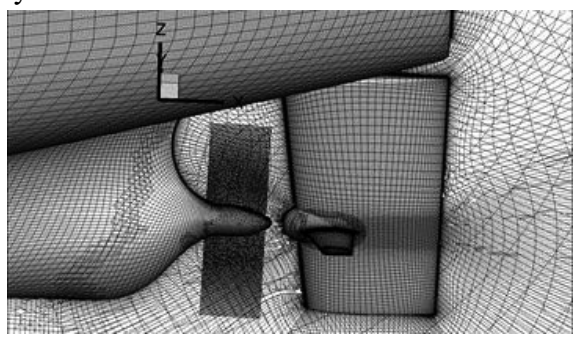

Fig. 2 Gird system around rudder with RBF

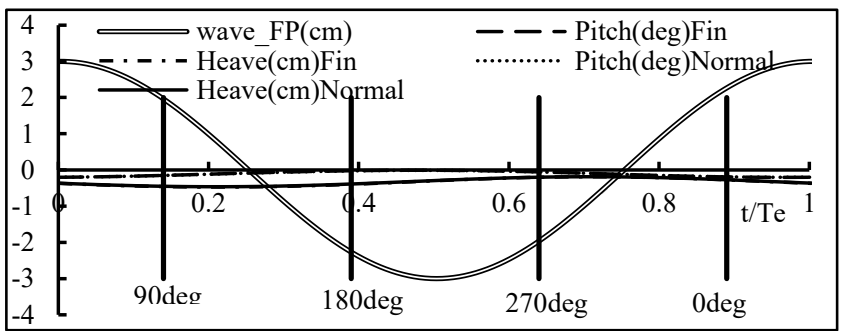

\section{2. 2 Computational domain and boundary conditions}

The non-dimensionalized computational domain extends as shown in Fig.3. The ship's forward perpendicular point and aft perpendicular point were set at $\mathrm{x} / \mathrm{L}=0$ and $\mathrm{x} / \mathrm{L}=1$. At the inlet, the wave calculated from the linear potential flow solution was generated. The boundary is far downstream so that the streamwise viscous effects are zero at the exit. The bottom and top of the background block were set as far-field boundary conditions. All the variables had zero gradient behavior at the sides of the domain. The surface of the ship hull and rudder was set as a no-slip boundary condition used for the surfaces which are moving with the grid.

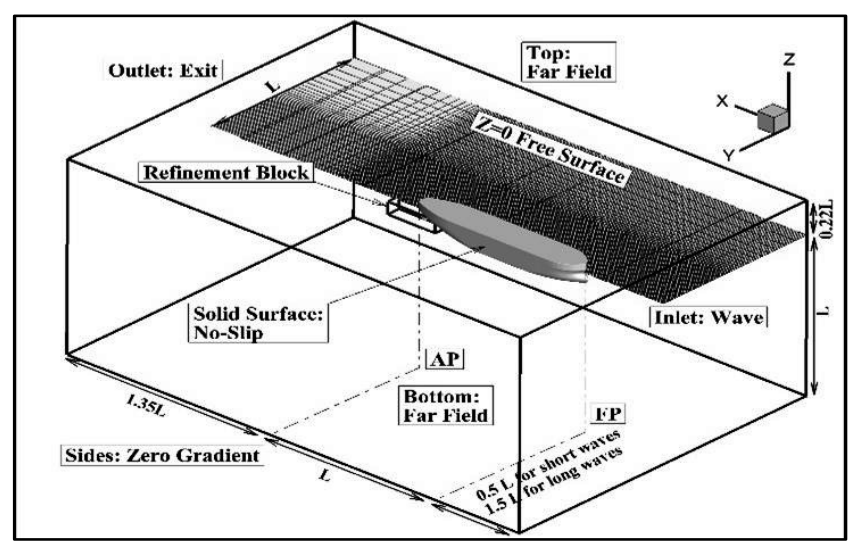

Fig. 3 Computational domain and boundary conditions

\section{Results and Discussion}

\section{1 Ship motion responses}

Firstly, the effect of ESD on the ship motions is investigated. In this paper, heave and pitch motion responses are discussed by comparing with and without ESD. Validation of CFD results with EFD data can be seen in Fig. 4 to Fig. 6. The time history of heave and pitch motion of two cases ( with normal rudder and with $\mathrm{RBF}$ )for one encounter period in $\lambda / \mathrm{L}=0.6,1.1$, and 1.6 are drawn in Fig. 4, Fig. 5, and Fig. 6 respectively, along with the incident wave data measured at the bow. The increase of the amplitude of motions with the increment of the wavelength was observed in all cases and both CFD and EFD. The fluctuation of both motions became the maximum in the largest wavelength, $\lambda / \mathrm{L}=1.6$. However, the mean values of motions that were around (minus) 0.3 $\mathrm{cm}$ in heave and (minus) 0.1 degree in pitch were quite the same in all wave conditions. The prominent changes in motions could not be investigated for all cases in both CFD and EFD as the lines of each motion coincided with each other. Hence, it can be confirmed that RBF does not have any effect on the ship's motion as it is quite small in size compared to the ship's size although it is located in the complex flow field behind the propeller. CFD can predict a similar trend of motions and waves measured by EFD.

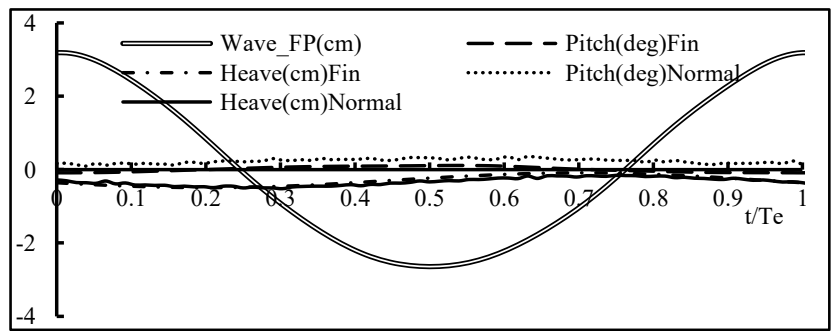

Fig. 4 Heave and pitch motions comparison at $\lambda / \mathrm{L}=0.6$ (Left: CFD \& Right: EFD) 

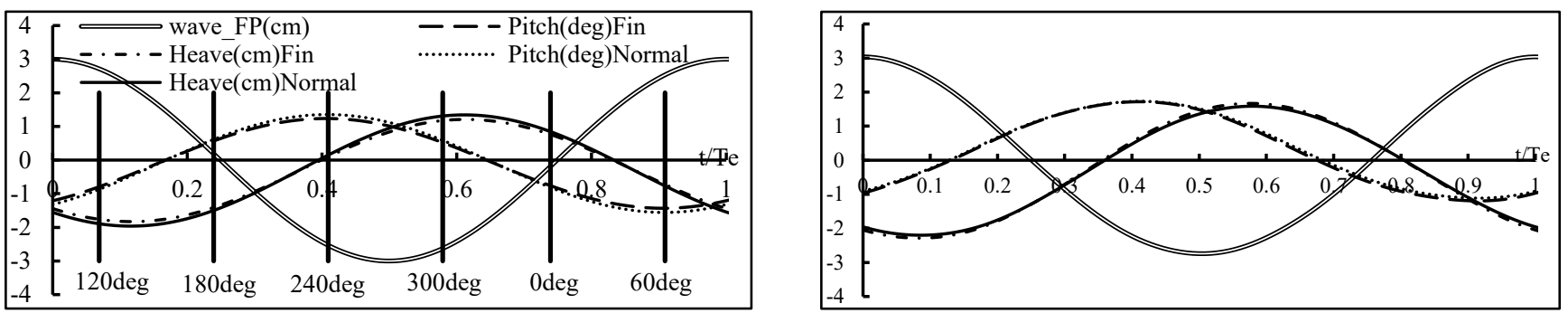

Fig. 5 Heave and pitch motions comparison at $\lambda / \mathrm{L}=1.1$ (Left: CFD \& Right: EFD)
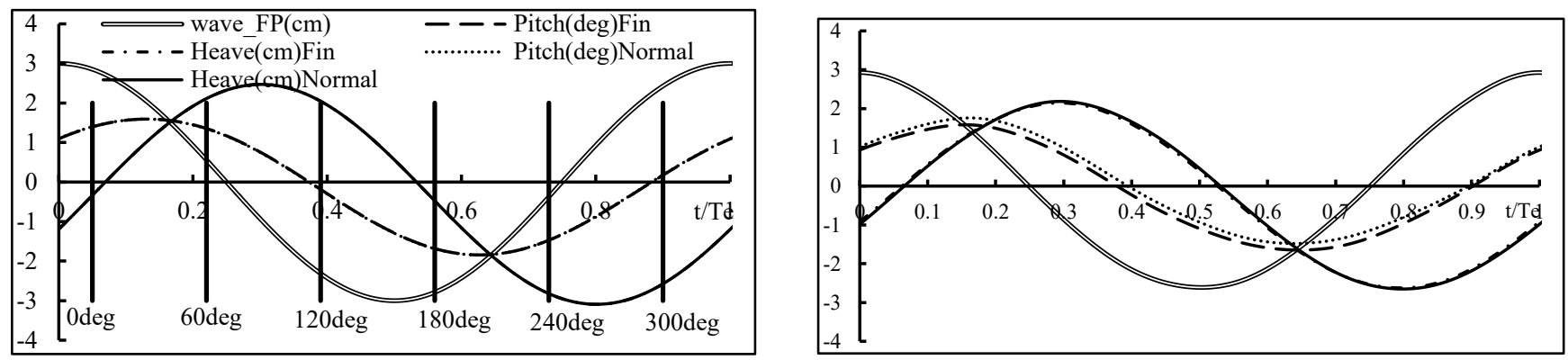

Fig. 6 Heave and pitch motions comparison at $\lambda / \mathrm{L}=1.6$ (Left: CFD \& Right: EFD)

\section{2 Flow field analysis}

\section{2. 1 Flow field visualization}

The measurement and analysis of the complex flow fields around the ship's stern play a vital role in the design of energysaving devices since the physical mechanism of ESDs can be fully understood from it. The measuring or computing positions were selected according to the configuration of the propeller and rudder. There were three positions in the longitudinal section along with the ship's length and one transverse position that is the centerline of a ship. One of the longitudinal positions was before the propeller and the others were at AP and after the rudder. Nevertheless, the flow field at only two longitudinal positions was measured in the experiment. All the measurements were carried out in four phases in short wave $(\lambda / \mathrm{L}=0.6)$ and six phases in medium and long waves $(\lambda / L=1.1$ and 1.6) for one encounter period as shown in Fig. 3-6. These phases were allocated by the wave data measured from the wave gauge meter installed $3.33 \mathrm{~m}$ ahead of the ship's bow. In this paper, the flow fields measured at two longitudinal positions and centre-plane of a ship in $\lambda / L=1.6$ are discussed.

To understand the detailed inflow characteristics of a propeller, Fig. 7 illustrates the flow field comparison between a ship with a normal rudder and with RBF at a non-dimensionalized distance of $\mathrm{x} / \mathrm{L}=0.96875$ which is about $4 \mathrm{~cm}$ before the propeller. The measurement shown in Fig. 7 was done at the zero degree phase which means the zero up-crossing point of the wave at wave gauge in $\lambda / \mathrm{L}=1.6$. X-coordinate is positive pointing towards the aft of the ship. Y-coordinate is positive toward starboard and $\mathrm{Z}$ is positive in upward direction. The $\mathrm{y}$ and $\mathrm{z}$ axes are non-dimensionalized by the ship length between the perpendiculars (Lpp). The wake field colored with non-dimensionalized axial velocity contour $(\mathrm{u} / \mathrm{Vs})$ is demonstrated together with the cross-flow velocity vector $(\mathrm{v} / \mathrm{Vs}$, $\mathrm{w} / \mathrm{Vs}$ ). $\mathrm{u}, \mathrm{v}$, and $\mathrm{w}$ are velocity components in $\mathrm{x}, \mathrm{y}$, and $\mathrm{z}$ directions. In all pictures, the boundary layer around the stern due to the friction of the hull and the hook-shaped vortex flow could be captured, note that the velocity is total velocity including propeller induced velocity. The wake fields in both port and starboard sides were almost symmetric. The flow tends to move down near the stern tube while moving upward far from the hull surface. In CFD simulation, low-speed contour and very small scale viscous vortex under the stern tube could be depicted in detail. The boundary layer thickness predicted by CFD was a little bit larger than that in EFD. There was not much difference in inflow velocity of the propeller between the normal rudder and RBF in both CFD and EFD.

The viscous wake dynamics downstream of the rudder is one of the primary concern of this research to see how much RBF can change and is effective. Measurement of the velocity field of a ship without a rudder case was also included to check the effect of a rudder on the stern's flow field. The comparison of the wake field fluctuation at $\mathrm{x} / \mathrm{L}=1.025$ that is $8 \mathrm{~cm}$ behind the AP for long wave can be seen in Fig. 8 . The measured phases were divided equally into six phases starting from 0 degree to 300 degree with an increment of 60 degree in one encounter period as in Fig. 6. According to the large motion of a ship in the long wave, movement of the velocity contour and transformation of its shape could be seen obviously in each phase degree. The downward flow was found in the starboard side of the rudder and the flow moved upward in the port side because of the clockwise rotation of the propeller. The circular vortex flow in a ship without a rudder was deformed and split into two main part when a rudder is fitted behind a ship. The propeller hub vortex could be observed in all figures. Theses vortex feature was not affected apparently while installing a normal rudder. However, its strength and velocity around its core became weak and small owing to the rudder bulb in the figures of the RBF case. The area of the wake field was larger in RBF compared to the normal rudder. These are some factors raising the hull efficiency that will be discussed later. The CFD captured well the flow field characteristic in all cases in the same way as EFD measurement. In CFD simulation, the dissipation of the vortex was a little bit higher due to the thin time averaged vortex ling sheet in NS code from short term averaged body force. The velocity in the downstream of the propeller was lower because the resolution of the grid in that region was relatively larger as compared with the propeller grid. That is why the flow field of the present computations was slower than the measured results.

Fig. 9 expresses longitudinally the axial velocity field at $\mathrm{y} / \mathrm{L}=$ 0 , a central plane of a ship in $\lambda / L=1.6$ computed by CFD. The direction of the flow followed the shape of the surface. The hub vortex moved and deformed to a shape that resembles that of the 
rudder profile (RBF or conventional rudder). This phenomenon, due to the effect of viscosity, is accompanied by diffusion of vorticity from the vortex filaments and that within the rudder boundary. It could be seen that the flow was accelerated to enhance

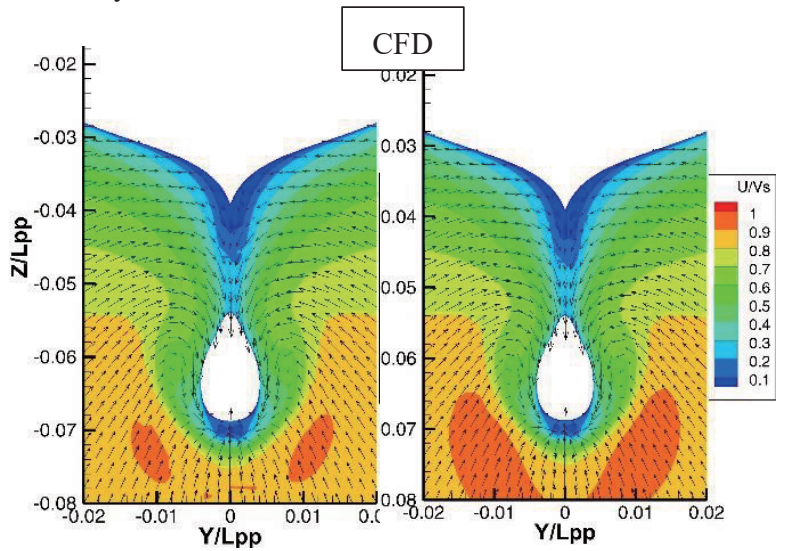

the effectiveness of the rudder while the rudder was behind the propeller slipstream. The relative expanded high-speed contour in RBF influenced hull efficiency.

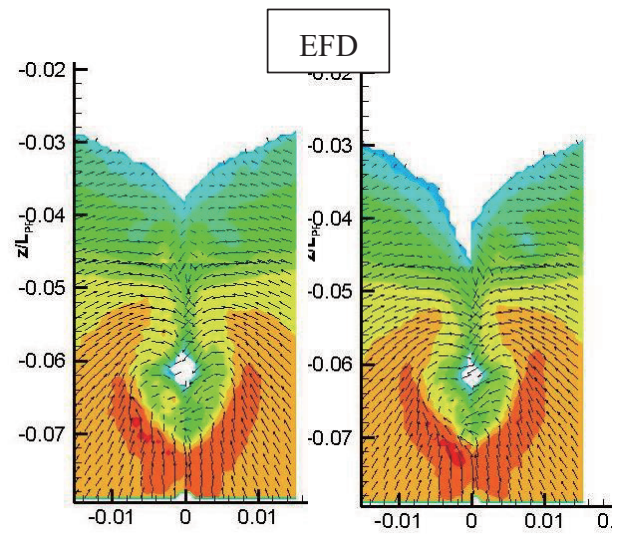

Fig. 7 Comparison of computed flow field and measured flow field at $\mathrm{x} / \mathrm{L}=0.96875$ in $\lambda / \mathrm{L}=1.6$ (Left: normal rudder and right: $\mathrm{RBF}$ )

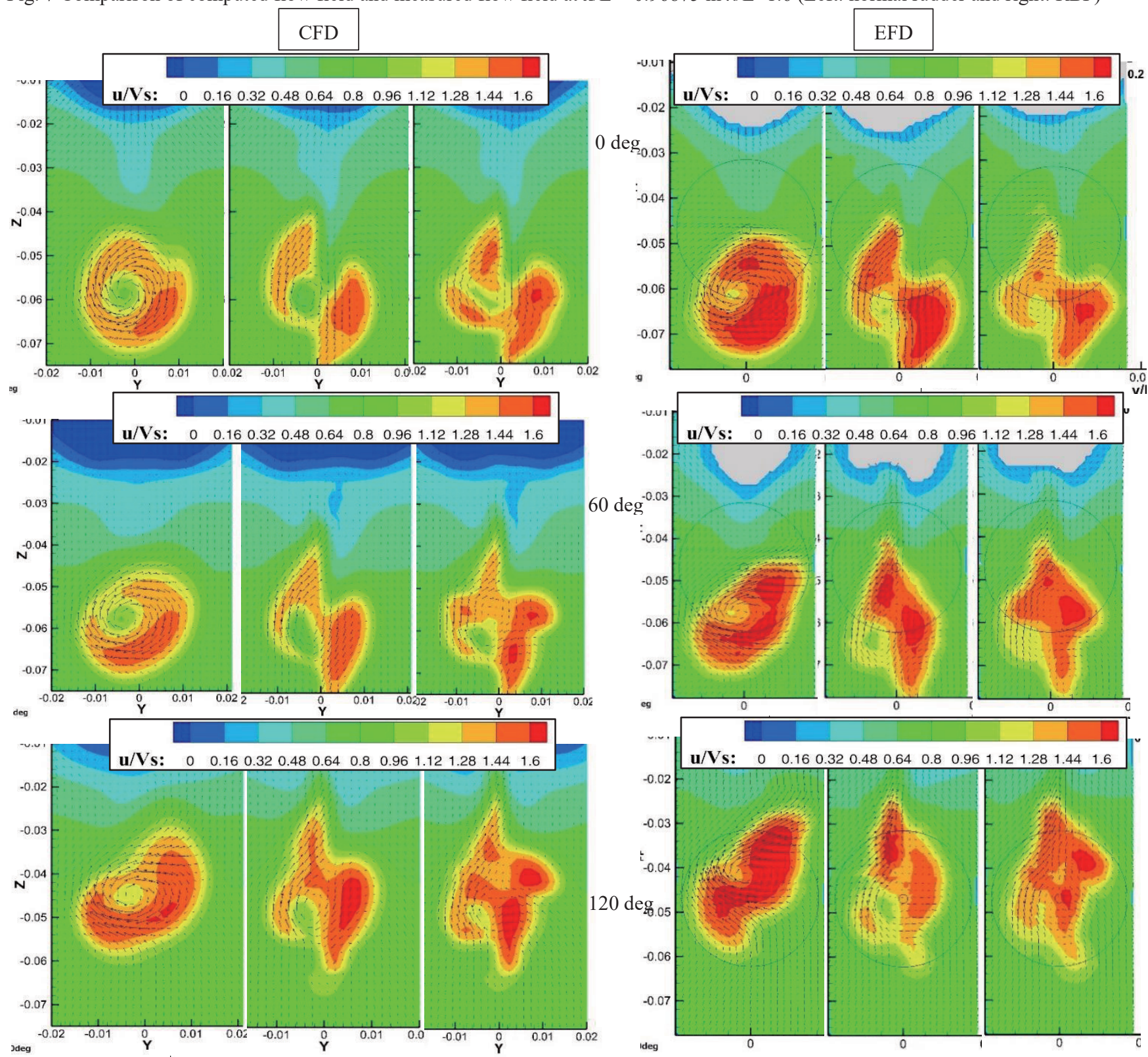

Fig. 8 Comparison of computed flow field and measured flow field at $\mathrm{x} / \mathrm{L}=1.025$ in $\lambda / \mathrm{L}=1.6$ (Left: no rudder, middle: normal rudder and right: RBF) 

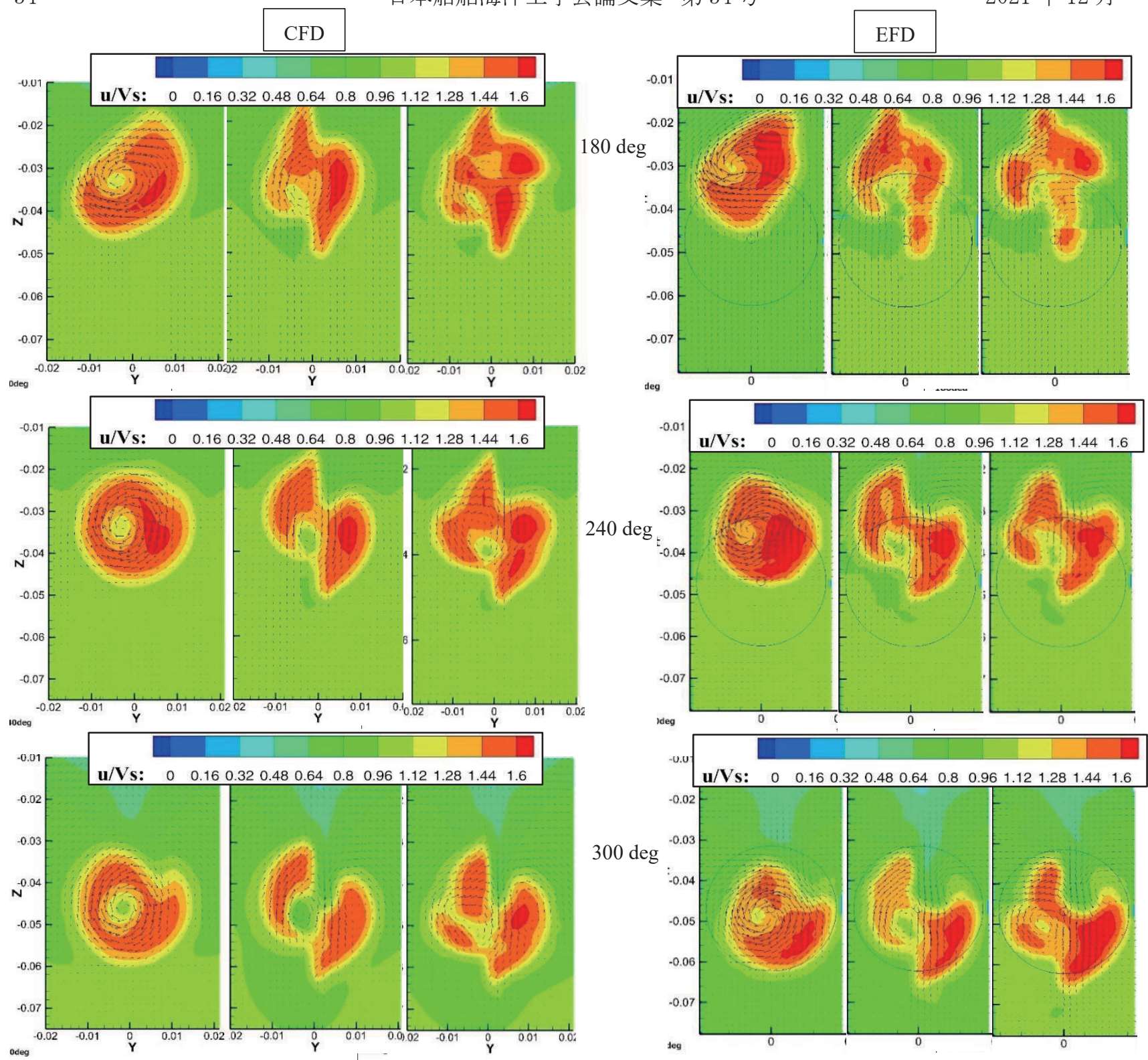

Fig. 8 (continued)
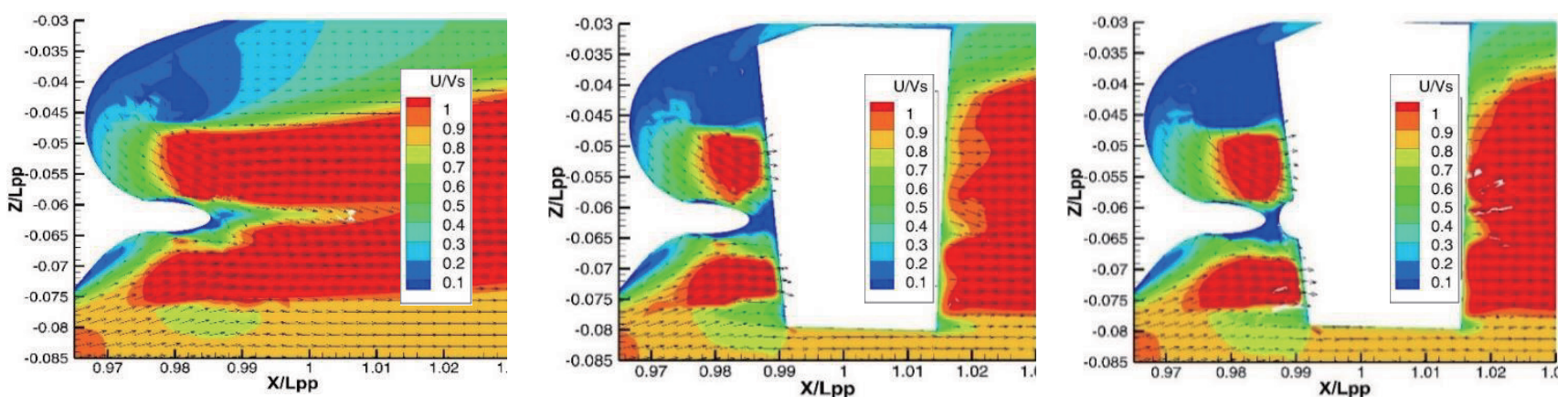

Fig. 9 Comparison of flow field computed by $\mathrm{CFD}$ at $\mathrm{Y} / \mathrm{Lpp}=0$ in $\lambda / \mathrm{L}=1.6$ (Left: no rudder, middle: normal rudder and right: $\mathrm{RBF}$ )

\section{2. 2 Time history of effective wake}

In this work, the thrust identity method was used to determine the effective wake coefficient. The thrust coefficient, $K_{T}$ is obtained as an output from the simulation, and then the advance number, $\mathrm{J}$ can be predicted on the propeller open water chart. The water inflow velocity into the propeller, $V_{A}$ was computed by the equation of $V_{A}=J(n D)$. The effective wake velocity, $V_{w}$ is the deduction of the inflow velocity from the full ship's speed. The effective wake fraction proposed by Taylor is computed by Eq. (6).
The time history of effective wake in the converged region is presented in Fig. 10. For the ship with one propeller, the wake fraction coefficient, $1-w$ is normally in the range of 0.8 to 0.55 . In this study, the average value of $1-w$ in all cases was over 0.5 which can be seen in Table 2 and Table 3. The CFD under-predicted the mean value of $1-w$ and computed a similar trend compared to the experiment. The effective wake was increased due to the rudder. It was increased more by rudder bulb and fins. Incidentally, a large wake fraction causes the risk of cavitation. That's why cavitation 
investigation of the propeller and the rudder should be performed while using RBF.

$$
1-w=\frac{V_{A}}{V_{S}}
$$

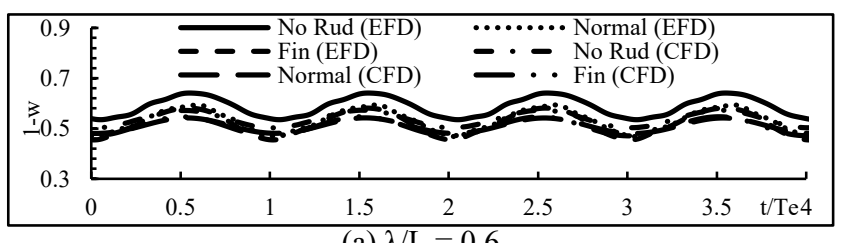

(a) $\lambda / \mathrm{L}=0.6$

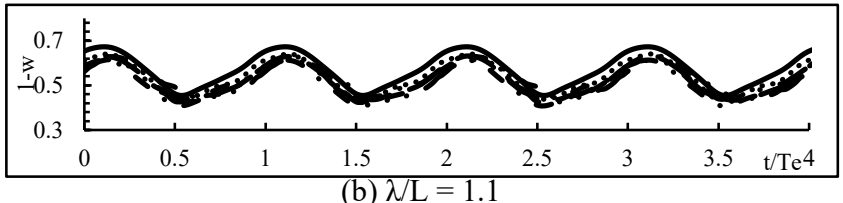

(b) $\lambda / \mathrm{L}=1.1$

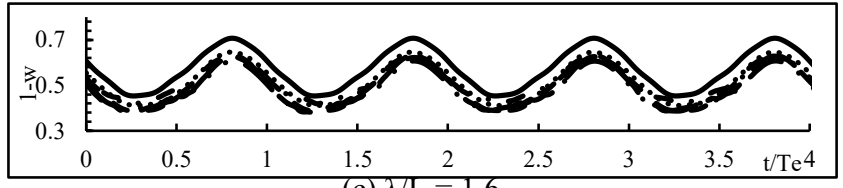

(c) $\lambda / \mathrm{L}=1.6$

Fig. 10 Comparison of time history of effective wake fraction

\subsection{Self-propulsion analysis}

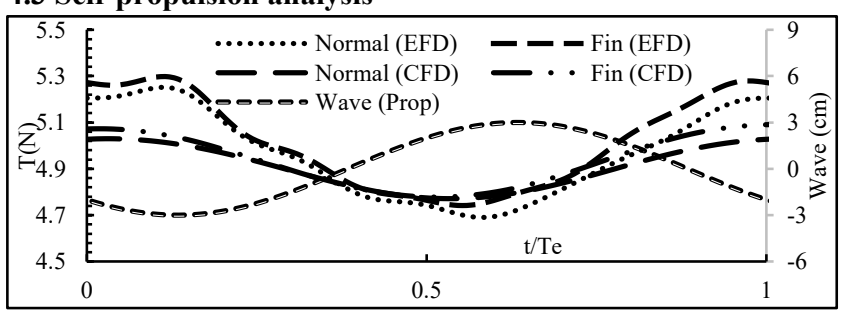

(a) $\lambda / \mathrm{L}=0.6$

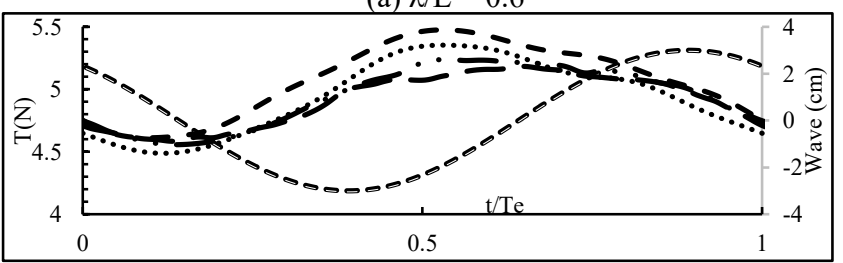

(b) $\lambda / \mathrm{L}=1.1$

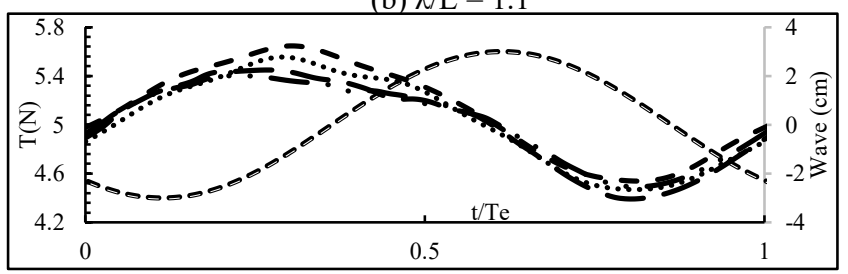

(c) $\lambda / \mathrm{L}=1.6$

Fig. 11 Comparison of time history of thrust

The efficient moving of a ship with low external force is one of the concerns that ship-owners and naval architects are demanding. For that reason, we examined the characteristic of resistance of a ship in waves and how much RBF influences it. In the CFD simulation, the total resistance coefficient, $C_{T}$ was computed by the summation of each and single resistance coefficient output. Then the total resistance of the ship, $R_{T}$ was calculated by Eq. (7). The convergence of the axial force time history curve was found after 3000 iterations. The time history of axial force in that converged region was extracted in four periods and presented in Fig. 12. Only the resistance data by CFD computation was included as the experimental data of the time history of resistance was not available. The resistance data oscillated in a simple harmonic pattern at $\lambda / \mathrm{L}=0.6$ and 1.6. But it was different in the medium wave where the resonance usually occurs. In the time history graph of axial force, any changes could not be found out between the normal rudder and RBF. However, as shown in Table2, it was observed that a rudder increased the resistance in the mean value. $\mathrm{RBF}$ reduced it in a small amount but it was still increased in contrast with the resistance of a ship without a rudder.

$$
R_{T}=0.5 C_{T} \rho S V_{S}^{2}
$$

where $\rho$ is the density of water and $S$ is the wetted surface area.

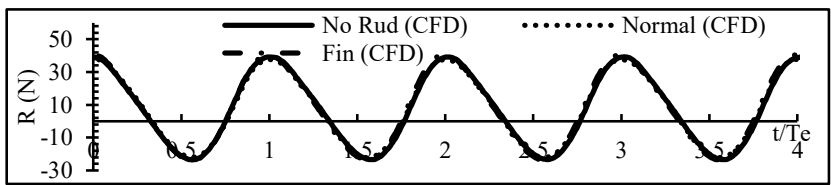

(a) $\lambda / L=0.6$

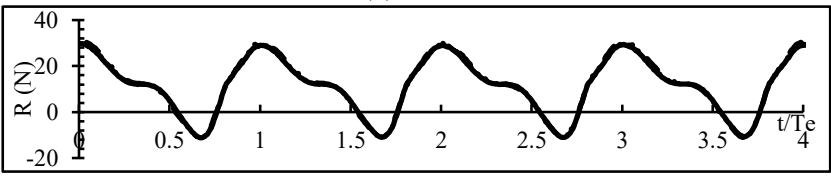

(b) $\lambda / \mathrm{L}=1.1$

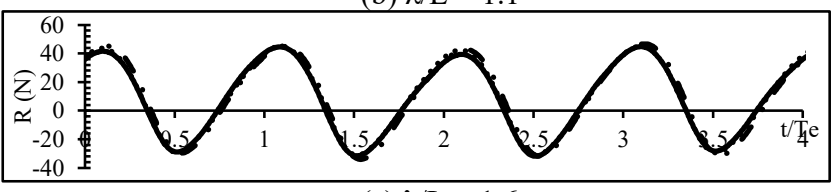

(c) $\lambda / \mathrm{L}=1.6$

Fig. 12 Comparison of time history of axial force

As shown in Eq. (8), the thrust was determined by the thrust coefficient, $K_{T}$ in the CFD computation. Fig. 11 gives the information about thrust time history comparison computed by CFD using 16.3rps and measured by experiment using 16.5rps. The thrust time history of all cases fluctuated harmonically in all waves. The fluctuation became large with increasing wavelength. When a rudder was fitted behind a ship, the thrust increased, and while mounting RBF, the thrust improved more in all wavelengths; revealing that $\mathrm{RBF}$ could generate the additional thrust by recovering the rotational flow behind the propeller. The CFD under-predicted thrust data in some cases and over-predicted in others. However, the error between CFD and EFD was quite small and was under $1.5 \%$.

$$
T=K_{T} \rho n^{2} D^{4}
$$

where $D$ is propeller's diameter which is $9.86 \mathrm{~cm}$ in this study.

The thrust deduction factor, $1-t$, was computed according to Eq. (9). In the equation, $R_{0}$ represents the towed resistance without a propeller in wave. The respective value of total resistance and thrust can be seen in Table 1 and Table 2. In a ship without ESD, normally the thrust deduction coefficient, $t$ should increase with increasing the wake fraction coefficient, $w$. It was obvious in Table 2 of comparison of a ship without a rudder and with normal rudder cases. However, the thrust deduction coefficient increased in some wavelengths due to the thrust increment when RBF was used. The value of the thrust deduction factor with one propeller normally is between 0.7 and 0.88 . The measured results of its value showed roughly above 0.8 since the block coefficient of KVLCC2 was fairly large.

The hull efficiency, $\eta_{H}$ can be defined as the ratio of the thrust deduction factor and effective wake fraction as Eq. (10). In other words, it is a relationship between effective power and propeller's thrust power. The average value of hull efficiency of a ship without 
rudder predicted by CFD was above 1.5 due to the high block coefficient although the normal hull efficiency is in the range of 1.1 and 1.4. When the normal rudder was installed downstream of the propeller, $\eta_{H}$ was imperceptibly decreased in short and long wavelengths. A small improvement was found in a ship with RBF; especially around $3 \%$ improvement at $\lambda / \mathrm{L}=1.1$ in both $\mathrm{CFD}$ and EFD. The CFD computational result of hull efficiency was reasonably close to the measured data by EFD with around 4\% error.

$$
\begin{gathered}
1-t=1-\left[\frac{R_{T}-R_{0}}{T}\right] \\
\eta_{H}=\frac{1-t}{1-w}
\end{gathered}
$$

Table 2 Self-propulsion factors by CFD

\begin{tabular}{|c|c|c|c|c|c|c|}
\hline $\begin{array}{c}\text { Waves } \\
(\lambda / \mathrm{L})\end{array}$ & $\begin{array}{c}\text { Rudder } \\
\text { Types }\end{array}$ & $\begin{array}{c}\mathrm{R} \\
(\mathrm{N})\end{array}$ & $\begin{array}{c}\text { Thrust } \\
(\mathrm{N})\end{array}$ & $1-\mathrm{t}$ & $1-\mathrm{w}$ & $\eta_{\mathrm{H}}$ \\
\hline \multirow{4}{*}{0.6} & No Rud & 7.3763 & 4.7759 & 0.8472 & 0.5408 & 1.5667 \\
\cline { 2 - 7 } & Normal & 7.4840 & 4.9028 & 0.7993 & 0.5109 & 1.5643 \\
\cline { 2 - 7 } & RBFS & 7.4143 & 4.9288 & 0.7920 & 0.5046 & 1.5697 \\
\hline \multirow{4}{*}{1.1} & No Rud & 11.4349 & 4.7865 & 0.8477 & 0.5378 & 1.5761 \\
\cline { 2 - 7 } & Normal & 11.9292 & 4.9138 & 0.8174 & 0.5157 & 1.5849 \\
\cline { 2 - 7 } & RBFS & 11.7944 & 4.9418 & 0.8190 & 0.4984 & 1.6433 \\
\hline \multirow{4}{*}{1.6} & No Rud & 7.7066 & 4.8992 & 0.8514 & 0.5109 & 1.6663 \\
\cline { 2 - 7 } & Normal & 8.3659 & 4.9378 & 0.7969 & 0.4926 & 1.6177 \\
\cline { 2 - 7 } & RBFS & 8.3603 & 4.9445 & 0.8076 & 0.5003 & 1.6142 \\
\hline
\end{tabular}

Table 3 Self-propulsion factors by EFD

\begin{tabular}{|c|c|c|c|c|c|c|}
\hline $\begin{array}{c}\text { Waves } \\
(\lambda / \mathrm{L})\end{array}$ & $\begin{array}{c}\text { Rudder } \\
\text { Types }\end{array}$ & $\begin{array}{c}\mathrm{R} \\
(\mathrm{N})\end{array}$ & $\begin{array}{c}\text { Thrust } \\
(\mathrm{N})\end{array}$ & $1-\mathrm{t}$ & $1-\mathrm{w}$ & $\eta \mathrm{H}$ \\
\hline \multirow{4}{*}{0.6} & No Rud & - & 4.7279 & - & 0.5878 & - \\
\cline { 2 - 7 } & Normal & 7.4287 & 4.9191 & 0.8331 & 0.5419 & 1.5374 \\
\cline { 2 - 7 } & RBFS & 7.4032 & 4.9273 & 0.8386 & 0.5416 & 1.5483 \\
\hline \multirow{4}{*}{1.1} & No Rud & - & 4.8039 & - & 0.5684 & - \\
\cline { 2 - 7 } & Normal & 11.1180 & 4.9247 & 0.8285 & 0.5423 & 1.5277 \\
\cline { 2 - 7 } & RBFS & 11.0768 & 4.9579 & 0.8379 & 0.5343 & 1.5682 \\
\hline \multirow{4}{*}{1.6} & No Rud & - & 4.8309 & - & 0.5624 & - \\
\cline { 2 - 7 } & Normal & 6.7486 & 4.9455 & 0.8362 & 0.5368 & 1.5576 \\
\cline { 2 - 7 } & RBFS & 6.8132 & 4.9822 & 0.8244 & 0.5287 & 1.5594 \\
\hline
\end{tabular}

\section{Conclusions}

The motion analysis, viscous flow field measurement at three positions and self-propulsion analysis were carried out to understand the working mechanism of RBF of the KVLCC2 ship model in regular head waves. A good agreement of computational results with the available experimental data was reported.

The RBF did not make the heave and pitch motions adverse in wave conditions. The motion characteristics of all cases (without rudder, normal rudder and RBF) were almost the same. The inflow velocity field of the propeller showed no difference between the normal rudder and RBF. The reduction of the hub vortex strength and enlargement of the wake field by RBF was found in the investigation of the flow field behind the rudder. The effective wake was gained due to RBF. In addition, the rudder fins produced additional forward thrust. The resistance was decreased by a small amount in certain wavelengths. The improvement of hull efficiency in waves was reported with $3.7 \%$ and $2.7 \%$ at medium wave in CFD and EFD, respectively. Unfortunately, it decreased around $1 \%$ in some wavelengths ${ }^{6}$. However, it improved favorably in calm water ${ }^{7}$. Thus, RBF can contribute to achieving the required EEDI index and meeting the goals of SDGs (Sustainable Development Goals).

To verify EEDI for ships with ESD's by classification companies, the extension of propulsive performance results from the model to full scale is necessary ${ }^{11)}$. The analysis in full scale is required be done to get more accurate results.

\section{Acknowledgement}

This work was partially supported by JSPS KAKENHI Grant Number $19 \mathrm{H} 02358$.

\section{References}

1) Tacar, Z., Sasaki, N., Atlar, M., and Korkut, E.: An investigation into effects of gate rudder system on ship performance as a novel energy-saving and manoeuvering device, Journal of Ocean Engineering, Vol. 218, 2020.

2) Okada, Y., Kawasaki, M., Katayama, K., Okazaki, A., and Fukuda, K.: The Development of Ultimate Rudder for EEDI, International Conference on Computational Methods in Maritime Engineering, Rome, 2015.

3) Su, Y., Lin, J., Zhao, D., Guo, C., and Guo, Hang.: Influence of a pre-swirl stator and rudder bulb system on the propulsion performance of a large-scale ship model, Journal of Ocean Engineering, Vol. 218, 2020.

4) Shen, H. L., Obwogi, E. O., and Su, Y. M.: Scale effects for rudder bulb and rudder thrust fin on propulsive efficiency based on computational fluid dynamics, Journal of Ocean Engineering, Vol. 117, pp. 199-209, 2016.

5) Htay, W. N., Truong, T. Q. and Toda, Y.: A CFD based Comparison Study of Conventional Rudder and Rudder with Bulb-Fins System of KVLCC2 in Waves, Proceedings of 30th ISOPE, Vol. 4, pp. 3377-3384, 2020.

6) Htay, W. N., Magari, A., and Toda, Y.: A Study on the Performance of Rudder Bulb Fins System of KVLCC2 in Regular Head Waves, Proceedings of 31 st ISOPE, Vol. 4, pp. 2643-2650, 2021.

7) Truong, T. Q., Wu, P-C., Aoyagi, K., Koike, K., Akiyama, Y. and Toda, Y.: The EFD and CFD Study of Rudder-Bulb-Fin System in Ship and Propeller Wake Field of KVLCC2 Tanker in Calm Water, Proceedings of $27^{\text {th }}$ ISOPE, 2017.

8) Mwangi, B. O., Matsuura, H., and Toda, Y.: The Effect of Energy Saving Devices (ESDs) on the Flow Field around the Stern of KVLCC2 Model Tanker, Proceedings of 31st ISOPE, Vol. 4, pp. 2651-2658, 2021.

9) Paterson, G., Wilson, W., and Stern, F.: General-purpose parallel Unsteady RANS Ship Hydrodynamics Code, CFDSHIP-IOWA, IIHR Report No.432, pp.2-21, 2003.

10) Tokgoz, E., Kuroda, K., Win, Y.N., and Toda, Y.: A New Method to Predict the Propeller Body-force Distribution for Modeling the Propeller in Viscous CFD Code without Potential Flow Code, Journal of the Japan Society of Naval Architects and Ocean Engineers, Vol. 19, pp.1-7, 2014.

11) Park, S., Oh, G., Rhee, S. H., Koo, B-Y., and Lee, H.: Full scale wake prediction of an energy saving device by using computational fluid dynamics, Journal of Ocean Engineering, Vol. 101, pp. 254-263, 2015. 